\title{
Analysis and Detection of Electrical Aging Effects on High-Speed Motor Insulation
}

\author{
Riccardo Leuzzi, Student, IEEE, Vito G. Monopoli, Senior, IEEE, Luca Rovere, Member, IEEE, \\ Francesco Cupertino, Senior, IEEE, and Pericle Zanchetta, Fellow, IEEE
}

\begin{abstract}
The use of fast wide-bandgap devices in high-speed electrical drives with steep pulse-width modulation voltage waveforms is the main cause of increased stress on the electric machine insulation system and, consequently, of reduced reliability of the whole system. This represents a major concern in safety-critical applications, such as in the aerospace and electric transportation fields. The novel contribution of this work is to assess the effects of electrical aging on complex insulation systems (i.e. a whole stator winding) by analyzing easily measurable macroscopic quantities. To this purpose, an electrical aging procedure is defined, and the effects produced on three tested motors are presented. Three different analysis methods are adopted with the aim of assessing the damage caused to an $\mathrm{AC}$ motor winding by such steep voltage waveforms and hence to evaluate the state of aging of the insulation system.
\end{abstract}

Index Terms - high-frequency converters, high-speed drives, insulation aging, partial discharges, pulse-width modulation.

\section{INTRODUCTION}

$\mathrm{O}$ VER the last years, high-speed electrical machines have been catalyzing the attention of the major manufacturers in the aeronautical and electric transportation fields since they allow a drastic reduction of system weight, size, and cost [1][3]. To be stably operated, such machines require high control and switching frequencies [4]. To this purpose, the use of highfrequency $(\mathrm{HF})$ converters with fast wide-bandgap power devices (e.g. SiC- and GaN-MOSFETs) is becoming a consolidated solution for high-speed drive applications [5], [6]. In addition to their fast dynamic performance, such devices present reduced switching energy losses with respect to the IGBT technology, allowing a reduction in the size of the heat dissipation systems, and thus to fully exploit the benefits of high-speed machines. However, the high-voltage gradients produced by the low switching times of these devices may become a cause of accelerated aging of motor insulation systems, leading to premature irreversible faults [7]. In fact, due to the steepness of the PWM voltage pulses supplying the

This paragraph of the first footnote will contain the date on which you submitted your paper for review. It will also contain support information, including sponsor and financial support acknowledgment. For example, "This work was supported in part by the U.S. Department of Commerce under Grant BS123456."

R. Leuzzi, V. G. Monopoli, and F. Cupertino are with the Department of Electrical and Information Engineering, Politecnico di Bari, Bari, Italy. (e-mail: riccardo.leuzzi@poliba.it,

francesco.cupertino@poliba.it)

L. Rovere, and P. Zanchetta are with the Department of Electrical and Electronic Engineering, University of Nottingham, Nottingham, UK. (e-mail: luca.rovere@nottingham.ac.uk, pericle.zanchettanottingham.ac.uk). electric machine, and because of the impedance mismatch between the motor and the cable that connects it to the converter, the cable may behave as a non-adapted transmission line. The voltage wave is continuously reflected across the cable, resulting in voltage overshoots and persistent oscillations at the machine terminals [8], [9]. Depending on cable length and PWM-pulse rise time, severe over-voltages may arise at the motor side, which amplitude can be more than twice the output voltage at the inverter side [10], [11]. Consequently, these phenomena are even more evident in high-speed electric drives because of the use of fast wide-bandgap devices.

Such overvoltage levels at the motor terminals are the triggering event for partial discharges (PDs), which are recognized to be one of the main causes of premature insulation breakdown in inverter-fed motors [12]. PDs are ionic discharge phenomena developing within the week spots of insulation systems (i.e. micro-cavities in the material or small air pockets resulting from non-perfect adhesion between the conductors) that slowly delaminate the dielectric material reducing its capability to withstand electric fields. Eventually, the material reaches a critical point where a total discharge incepts, leading to the final breakdown [13], [14]. In [15] the relationship between PDs and the breakdown behavior of typical turn insulation is illustrated, and a correlation between the amplitude of the PWM pulses and the number of pulses to breakdown is drawn. Further studies are conducted in [16] on the effects of the PWM-pulse features (i.e. rise time, frequency, pulse duration, etc.) on the PD characteristics (i.e. magnitude, repetition rate, delay time, etc.). The expected lifetime of an insulation system as a function of the rise time and frequency of the pulses is also studied. In [17] the inter-turn electrical stress is analyzed demonstrating how the interaction between the short voltage rise time and the parasitic capacitances introduced by the insulation system produce an uneven voltage distribution along the coils. As a result, during the first instants after the application of a pulse, a large share of the voltage concentrates on the first coils, further increasing the stress for the insulation.

Therefore, it is clear that low values of rise time and high values of switching frequency lower the drive reliability by reducing the insulation system lifetime. This represents a major concern in all those fields where the continuity of operation is of the utmost importance, for either safety (e.g. aerospace or ground transportation areas) or economic reasons (e.g. industry).

In this scenario, the aim of this paper is to investigate the 
effects of high-frequency, steep PWM voltage waveforms on the degradation of the winding insulation system of randomwound AC motors. The innovative contribution consists in the original approach to this kind of analysis. The approaches employed in the literature in this field, in fact, can be roughly classified into two types. A first group of papers focuses the analysis on the microscopic effects produced by PDs on very simple systems (i.e. twisted magnetic wires or insulation bulks with defects of known geometry), in which a single discharge phenomenon is more easily observable and its physical and chemical features measurable [15], [16], [18]. On the other hand, a second group presents the analysis of macroscopic effects produced on complex systems (i.e. from single highvoltage motor bars to entire random-wound stators) by thermal aging only [19], [20]. Finally, [21] propose an off-line analysis method that is tested by simulating the aging of the machine. None of the previous works has carried out an analysis of the macroscopic effects produced on complex systems, such as random-wound motor windings, by electrical aging. This work presents the results of an accelerated electrical aging campaign on three random-wound motors showing how it can affect some macroscopic quantities. The aim of the work is not to bring the system to breakdown, but to investigate the role of the abovedescribed phenomena in a realistic application, such as a motor fed by the HF PWM voltage pulses.

To this purpose, different methods are adopted in this paper to study the consequences of such steep PWM waveforms on the health status of a random-wound winding. In particular, since the parasitic capacitive nature of the winding insulation is predominant to the inductive behavior of the machine in the high-frequency domain, the presented approaches focus on the $\mathrm{HF}$ characteristics of the machine. The first method analyzes the variation of the HF characteristic impedances of the machine under test (MUT). A second approach focuses on the observation of the HF components of the phase current and its frequency spectrum. The advantages and downsides of the different approaches are then highlighted to compare the capability to detect the progressive damage caused on the motor winding insulation by the aging campaign. Although this paper is based on the work presented in [22], an innovative contribution is provided in terms of a more effective definition of the diagnostic tools and of many more presented experimental results that prove the effectiveness of the proposed solution.

The paper is organized as follows. In Section II, the accelerated electrical aging procedure and the experimental setup are described. The approaches employed to assess the state of aging of the machine winding are presented in Section III. The experimental results are presented and discussed in Section IV and conclusions are drawn in Section V.

\section{EXPERIMENTAl Setup AND ACCELERATED AgING TEST}

The machines tested in this study are three identical lowvoltage, general-purpose induction motors, whose rated parameters are listed in Table I. A schematic representation of the experimental bench is depicted in Fig. 1. It consists of a SiCMOSFET inverter connected to the MUT through a 30-meter-
TABLE I

RATED PARAMETERS OF THE MOTORS UNDER TEST

\begin{tabular}{lcc}
\hline \hline \multicolumn{1}{c}{ Parameter } & Unit & Value \\
\hline Output power & $\mathrm{kW}$ & 0.37 \\
Full load speed & $\mathrm{rev} / \mathrm{min}$ & 1370 \\
Voltage $(\mathrm{Y} / \Delta)$ & $\mathrm{V}$ & $230 / 400$ \\
Rated current $(\mathrm{Y} / \Delta)$ & $\mathrm{A}$ & $1.93 / 1.11$ \\
Locked rotor current & $\mathrm{p.u}$. & 3.6 \\
Full load torque & $\mathrm{Nm}$ & 2.58 \\
Insulation class (temp. rise) & $-(\mathrm{K})$ & $\mathrm{F}(80)$ \\
\hline \hline
\end{tabular}

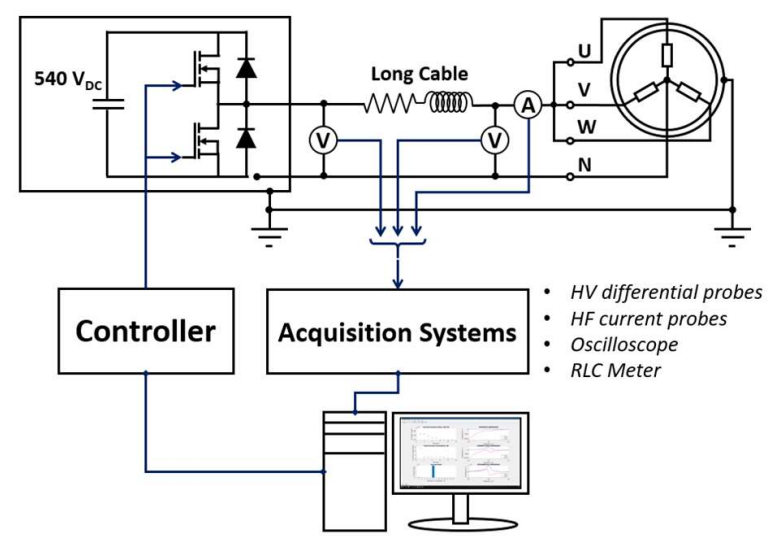

Fig. 1. Schematic structure of the experimental bench employed for the electrical aging procedure.

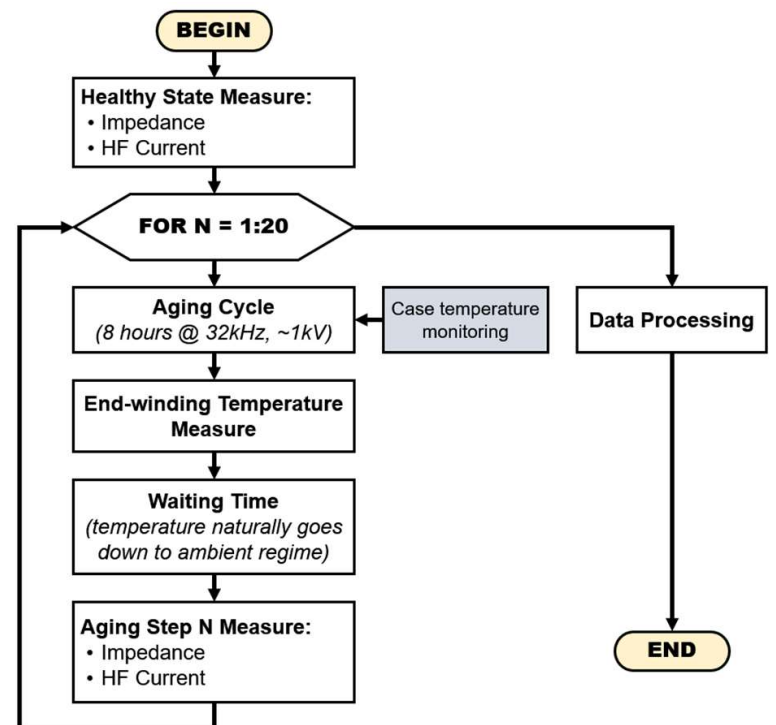

Fig. 2. Flow chart detailing the routine followed for the tests.

long cable to magnify the voltage reflections at the motor terminals, a controller to drive the inverter gates, a PC to handle the tests, and various system to perform acquisition of voltage, currents, temperature, etc. Fig. 2 shows a flow chart detailing the steps followed for the aging tests. The first step of an aging campaign on a MUT is the measurement of its HF characteristics in the healthy state, which will be then used as a reference for the estimation of the aging effects. Then, the first aging cycle is started. The MUT is a random-wound machine rated $0.37 \mathrm{~kW}$, insulation class $\mathrm{F}\left(155^{\circ} \mathrm{C}\right)$, connected in star configuration with accessible neutral. During the aging 
procedure, the inverter is operated with a DC-link voltage of $540 \mathrm{~V}$ and a switching frequency of $32 \mathrm{kHz}$. The PWM pulses are applied between the motor phase terminals connected in parallel and the neutral (i.e. the three-phase windings are connected in parallel). Each aging cycle lasts for 8 hours, thus a total of $921.6 \cdot 10^{6}$ pulses are applied to each phase during each cycle. The voltage waveforms at the inverter output and at motor terminals are shown in Fig. 3a. The rise time of the inverter voltage pulse is around $70 \mathrm{~ns}$, producing a peak overvoltage on the motor of $980 \mathrm{~V}$. After the aging cycles terminates, the MUT is left unsupplied for one night, in order to let the temperature naturally reach the ambient regime. The measurement procedure to register the HF characteristics of the machine after the aging step is performed in the morning. The details about the measurements are given in Section III. Then, a new aging cycle is started and the routine repeats until the selected number of cycles is reached.

To avoid undesired thermal effects that could affect the results of the study, it is important to ensure that the temperature of the motor windings lays well below the critical value of the insulating material (i.e., $155^{\circ} \mathrm{C}$ in this case). For this reason, a few measures are taken to prevent that thermal damage overlaps to the effects of the electrical discharges during the procedure. First, the aging cycles are performed at a constant duty cycle equal to $7 \%$ in order to limit the RMS current absorbed by the motor below the rated value. Moreover, the MUT is externally cooled with forced ventilation to improve heat dissipation. The temperature of the motor case is regularly monitored during the aging procedure using a thermographic camera. After a few hours of aging, the case temperature is around $40{ }^{\circ} \mathrm{C}$, as shown in Fig. 3b. It has been verified that in this condition the winding temperature is around $55-60^{\circ} \mathrm{C}$, well below the critical limit of the material. As an additional verification, the end-winding temperature is measured after two minutes from the end of each aging cycle, after removing the case front closure. Its value is always around $55^{\circ} \mathrm{C}$.

\section{MEthods OF ANALYSIS}

As stated before, the parasitic capacitances introduced by the insulation system of the machine dominate its inductive behavior in the high-frequency domain. These capacitances are determined by the structure and properties of the insulating material between the conductors, or between the conductors and the stator iron, which is grounded. As a consequence, a degradation of the insulation properties affects the equivalent value of the parasitic capacitances and can be observed in the machine high-frequency behavior. To this purpose, after each electrical aging cycle, the effects of the electrical stresses on the motor winding insulation are investigated by analyzing its HF characteristics. These characteristics are evaluated from the measurement of the response both in the frequency and in the time domains, i.e. by acquiring the motor characteristic impedances as a function of the frequency, and by analyzing the current response to a steep voltage pulse applied between the phases.

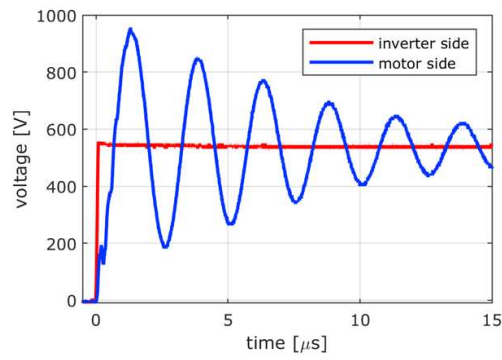

(a)

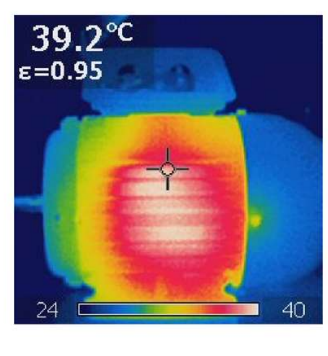

(b)
Fig. 3. Experimental overvoltage at motor side (a) and temperature on the motor case during an aging cycle, measured after a few hours from cycle start (b).

\section{A. High-Frequency Characteristic Impedances}

An impedance analyzer has been employed to measure the machine impedance in a wide frequency range. It can span a frequency range between $100 \mathrm{~Hz}$ and $40 \mathrm{MHz}$, with high accuracy and repeatability of the measures. Three characteristic impedances can be defined according to the relative connection of the machine terminals to the instrument for the measure. The common mode impedance is measured between the three phase terminals shorted together and the ground terminal, while the neutral is floating. The differential impedance is measured between the three phase terminals shorted together and the neutral, while the ground terminal is floating. Finally, the parasitic impedance is measured between the three phases and the neutral shorted together and the ground terminal. The common mode, differential, and parasitic impedances of one of the MUT as a function of frequency are shown in Fig. 4, 5, and 6 , respectively. For each figure, the healthy insulation state and the measures taken after a few aging cycles are compared.

The characteristic impedances of an electric machine have typical shapes, with the relevant information being generally contained in the frequency range between $10 \mathrm{kHz}$ and $1 \mathrm{MHz}$. At low frequency, the parasitic and common mode impedances have a highly capacitive nature (the phase is near to $-\pi$ ), with large magnitude mainly due to the effect of the insulation that blocks current dispersion from the winding to the motor case. With the frequency increasing, the imaginary part of the impedance decreases sharply until, around $100 \mathrm{kHz}$, a resonance peak is determined by the interaction with the coil inductance in the common mode path of the current. At the very high frequencies, the behavior is again highly capacitive, due to the fact that the turn-to-turn and the turn-to-ground capacitances are the minimum impedance path for the HF currents. The differential impedance has, instead, an ohmicinductive behavior at low frequency, which becomes capacitive after the resonance peak, when the turn-to-turn parasitic capacitances become the minimum impedance path for current circulation. It is worth noticing that the differential impedance is the one that the inverter actually "sees" in each switching condition. In each of its states, in fact, the switches are always set so that a phase of the motor is in series with the parallel of the other two.

As can be seen in Fig. 4, 5, and 6, with the progression of the insulation aging, the amplitude and position of the resonance peak slightly change. Therefore, it is possible to calculate an 
index that measures this displacement after each aging cycle to track the effects of the electrical stress on the insulation. The index employed in this paper is calculated as the root mean square deviation (RMSD) between the healthy state and the considered aging state. It is referred to as impedance deviation and denoted as $d_{z}$.

$$
d_{z, j}=\sqrt{\frac{1}{n} \sum_{k=1}^{n} w(k) \cdot\left[Z_{0}(k)-Z_{j}(k)\right]^{2}}
$$

In (1), $n$ is the number of measurement points, $Z_{0}(k)$ is the module of the healthy state impedance at the frequency $k$, and $Z_{j}(k)$ is the module of the impedance after $j$ aging cycles at the frequency $k . w(k)$ is a weighting function with values between 0 and 1 that is used to limit the calculation to the frequencies of interest (in this case, it is a rectangular function with value 1 between $10 \mathrm{kHz}$ and $1 \mathrm{MHz}$ ).

A second version of this index can be defined as the RMSD between an aging state and the previous one, i.e. substituting $Z_{j-1}(k)$ for $Z_{0}(k)$ in (1). The information given by this index can be useful to highlight the entity of the change between two consecutive cycles, regardless of the direction, and is therefore referred to as differential impedance deviation and denoted as $d_{z}^{D}$. Finally, an integral impedance deviation $\left(d_{z}^{I}\right)$ can be calculated as the sum of the differential deviations after each aging cycles and can be useful to track the evolution of the impedance with the time.

\section{B. High-Frequency Currents Components}

Generally speaking, the steepness of the PWM voltage pulses excites the high-frequency modes of the armature winding. As a result, the current that the motor absorbs in response to a single voltage pulse presents an initial high-frequency transient superimposed to the slope proportional to the machine lowfrequency reactance. In order to capture this high-frequency transient, the current of one phase is recorded by mean of a Rogowski coil connected to a GPIB-programmable digital oscilloscope. The current probe that has been employed has a peak di/dt of $20 \mathrm{kA} / \mu \mathrm{s}$ and a bandwidth of $20 \mathrm{MHz}$. The Rogowski probe is positioned inside a shielded box to prevent interference during the measure. Its position is fixed in the box in order to acquire the current response in the same position during each measure. 50 scores are taken for each aging state with the purpose of making some statistical considerations on the results. For each score, a voltage step of $540 \mathrm{~V}$ is applied to the winding of the machine and maintained for about $80 \mu \mathrm{s}$. The rising flank of the voltage step triggers the acquisition on the oscilloscope, and the current data points are recorded in a window of $50 \mu \mathrm{s}$ at a sample rate of $500 \mathrm{MS} / \mathrm{s}$. The trigger delay is set to $5 \mu \mathrm{s}$, so that an adequate number of samples around zero current is available to correct the acquired data from the offset of the probe. As a result, the net observation window useful for the high-frequency transient analysis is equal to 45 $\mu$ s. Fig. 7 shows a schematic timing diagram of the measuring procedure of one score. Considering the very short duration of the applied voltage pulse and the relatively large time required
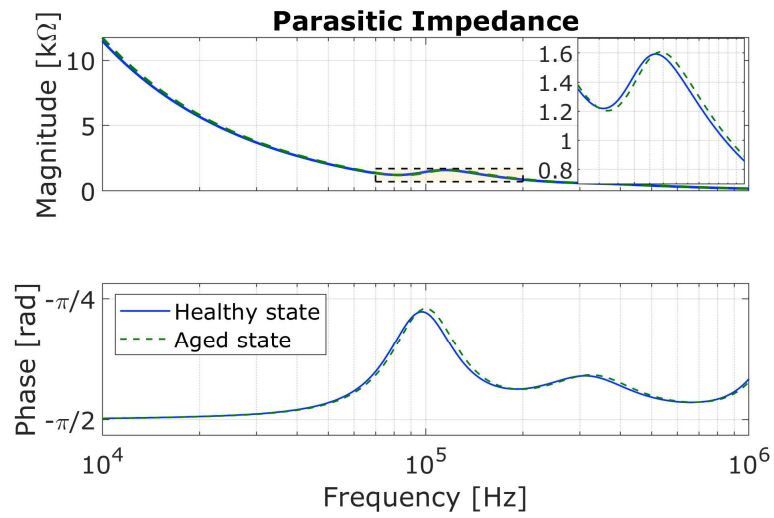

Fig. 4. Parasitic impedance: comparison between healthy and aged insulation state.
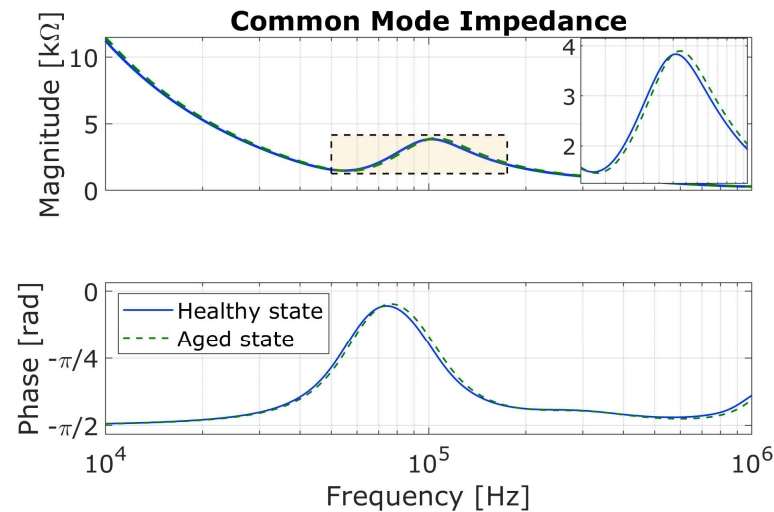

Fig. 5. Common mode impedance: comparison between healthy and aged insulation state.
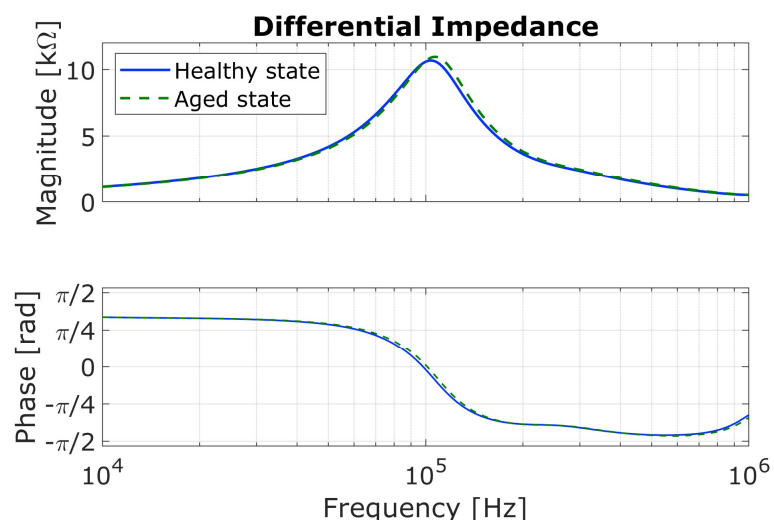

Fig. 6. Differential impedance: comparison between healthy and aged insulation state.

to transfer the acquired data samples from the oscilloscope to the PC (around $10 \mathrm{~s}$ in total), it can be assessed that no temperature variations affect the outcome of the measure during this procedure.

The current waveforms averaged over the 50 scores, for the healthy and for an aged insulation state are shown in Fig. 8, which also includes a detail of the first $1 \mu$ s from the application of the voltage step. The high-frequency transient changes as the motor undergoes the aging process, are related to the capacitive behavior of the motor, and thus to the insulation properties. For this reason, the deviation of the current from the healthy state of the machine can be used to derive an index of the degradation 


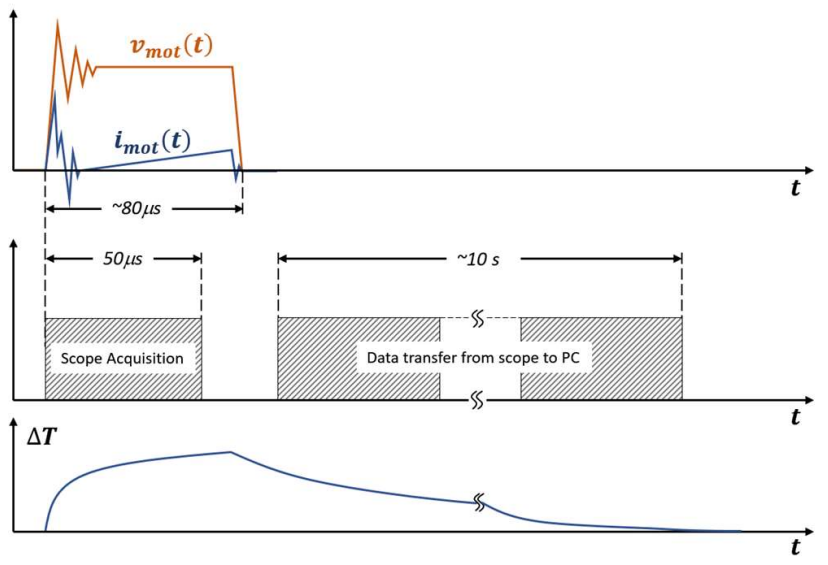

Fig. 7. Schematic timing diagram of the high-frequency current measurement procedure.

of the insulation, similarly to what is done with the characteristic impedances. The current deviation $d_{i}$ can be calculated, for each aging step, as the average RMSD with respect to the healthy state

$$
d_{i, j}=\operatorname{mean}_{s}\left\{\sqrt{\frac{1}{n} \sum_{k=1}^{n} w(k) \cdot\left[i_{0}^{a v g}(k)-i_{j, s}(k)\right]^{2}}\right\}
$$

where $n$ is the number of samples of the current waveform, $i_{0}^{a v g}(k)$ is the $k^{\text {th }}$ sample of the current at the healthy state, averaged over the total number of scores, and $i_{j, s}(k)$ is the $k^{\text {th }}$ sample of the score $s$ of the current acquired after $j$ aging cycles. $w(k)$ is a weighting function with values between 0 and 1 that is used to limit the calculation to the time interval of interest (in this case, it is a rectangular function with value 1 between 0 and $25 \mu \mathrm{s}$ ). As for the impedance deviation, it is also possible to define a differential current deviation $\left(d_{i}^{D}\right)$ and an integral current deviation $\left(d_{i}^{I}\right)$ to highlight the entity of the variation regardless of its direction.

In order to gain a better visualization of the variation in the frequency domain, a Fast Fourier Transformation (FFT) algorithm is used. Fig. 9 shows the spectra obtained from the same aging states that are shown in Fig. 8, averaged over the 50 scores. The major difference caused by the aging process occurs in the range from $500 \mathrm{kHz}$ to $10 \mathrm{MHz}$, confirming the results observed in the current transient.

Like the high-frequency currents, also their spectra can be compared to evaluate an index of the insulation degradation. This index, called insulation state indicator (ISI), was firstly proposed by C. Zoeller et al. in [19] for diagnosis purposes. In this paper, in accordance with the other indices proposed, it will be referred to as spectrum deviation $\left(d_{Y}\right)$. Its calculation is as in (3).

$$
d_{Y, j}=\operatorname{mean}_{s}\left\{\sqrt{\frac{1}{n} \sum_{k=1}^{n} w(k) \cdot\left[Y_{0}^{a v g}(k)-Y_{j, s}(k)\right]^{2}}\right\}
$$

Here, $n$ is the number of harmonics of the current spectrum, $Y_{0}^{a v g}(k)$ is the $k^{\text {th }}$ amplitude of the current spectrum at the

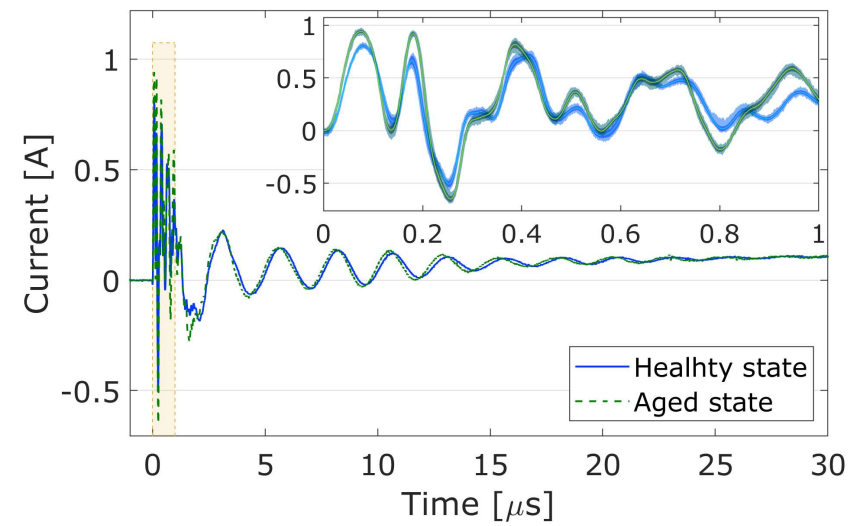

Fig. 8. Current in phase $U$ measured after applying a voltage step for two different aging states. The detail shows the maximum and standard deviations of the measure.

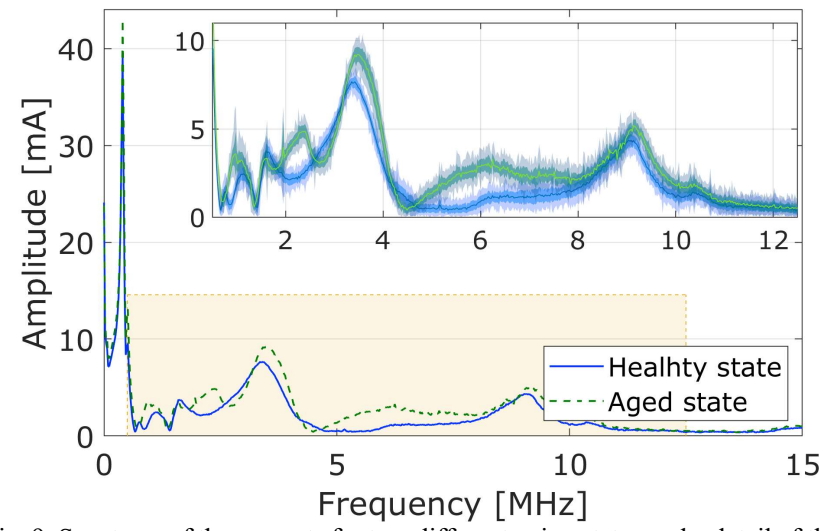

Fig. 9. Spectrum of the currents for two different aging states and a detail of the frequency range of interest for the calculation of the deviation.

healthy state, averaged over the total number of scores, and $Y_{j, s}(k)$ is the $k^{\text {th }}$ amplitude of the current spectrum of score $s$, acquired after $j$ aging cycles. $w(k)$ is a weighting function with values between 0 and 1 that is used to limit the calculation to the frequency interval of interest (in this case, it is a rectangular function with value 1 between $500 \mathrm{kHz}$ and 10 $\mathrm{MHz}$ ). It is important to properly select the calculation window because, above a certain frequency, the spectrum is influenced by the measurement noise of the probe, which could lead to a poor interpretation of the results. Moreover, before calculating the FFT on the current signal, it is necessary to remove the constant inductive slope that can be noticed in Fig. 8, since it affects the correctness of the results. A differential spectrum deviation $\left(d_{i}^{D}\right)$ and an integral spectrum deviation $\left(d_{i}^{I}\right)$ are also defined, as for the previous cases.

In order to verify that the variation observed in the highfrequency current transient is actually due to the effects of aging, the maximum and standard deviation bands are also depicted in Fig. 8 for both aging steps. As it can be noticed from the figure, the measurement accuracy and repeatability are quite good, thus letting a proper comparison of the scores with the purpose of quantifying the insulation degradation. Fig. 10 shows the distribution of all the samples of HF current that have been acquired, normalized with respect to their mean values. The standard deviation calculated considering all the samples is equal to $35 \mathrm{~mA}$, thus confirming the goodness of the measure. 


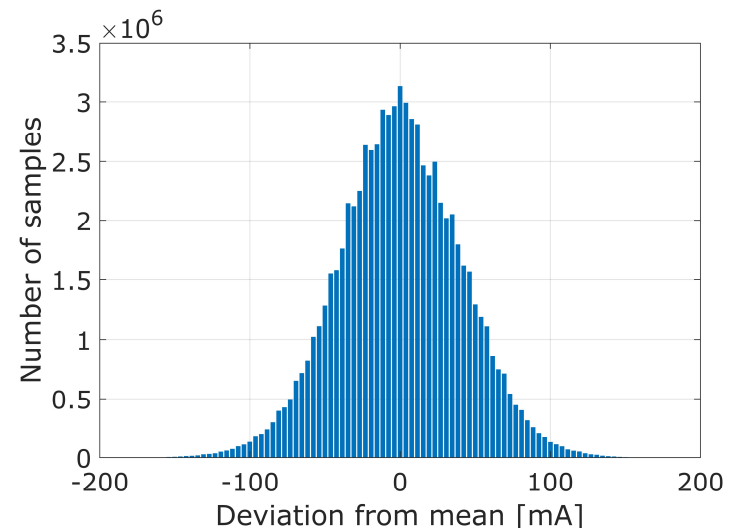

Fig. 10. Distribution of the sample data of the high-frequency current, normalized with respect to their mean value.

The same analysis has been performed on the FFT data, obtaining a standard deviation value of about $3 \mathrm{~mA}$. This verification confirms that the measures are accurate enough to discern small variations on the HF characteristics.

\section{RESULTS AND DISCUSSION}

This section presents the results of the aging campaign conducted on the three MUTs according to the measuring procedures and to the index calculations illustrated in the previous sections. Fig. 11, Fig. 12, and Fig. 13 show the impedance, current, and spectrum deviations as a function of aging time. For each figure, the base, differential, and integral versions of the indices are reported. It is worth highlighting the two different perspectives underlying the definition of the three versions of each index. After each aging cycle, the base version of the indices defined according to (1), (2), and (3) evaluates the actual insulation aging state as the absolute distance from the healthy state, regardless of the path followed to reach that state. In fact, it has been observed that the measured macroscopic quantities could undergo negative variations as well. Consequently, the base deviations are characterized by an up-and-down behavior, as it can be noticed in the uppermost plot in the figures. A negative variation of the index, and hence a reduction in the distance from the healthy state, should not be directly interpreted as an insulation recovery phenomenon. The electrical aging process on a complex system, such as the stator winding, produces alterations of the insulation condition that are characterized by randomness. In fact, casual modifications can happen in different parts and with different intensity within the system. The cumulative effect of such random variations determines the overall capacitive behavior that can be observed through measuring macroscopic quantities. It is for this reason that the differential and integral deviations are introduced for each index. The differential deviation, in fact, provides information about the distance between two consecutive aging steps, thus putting the focus on the intensity of single variations. Being carried out as the sum of the differential deviations at each aging step, the integral deviation can reconstruct the overall path of aging that the insulation system experiences, still giving information with respect to the starting point (i.e. the healthy state).

In Fig. 11, the base impedance deviation presents an initial
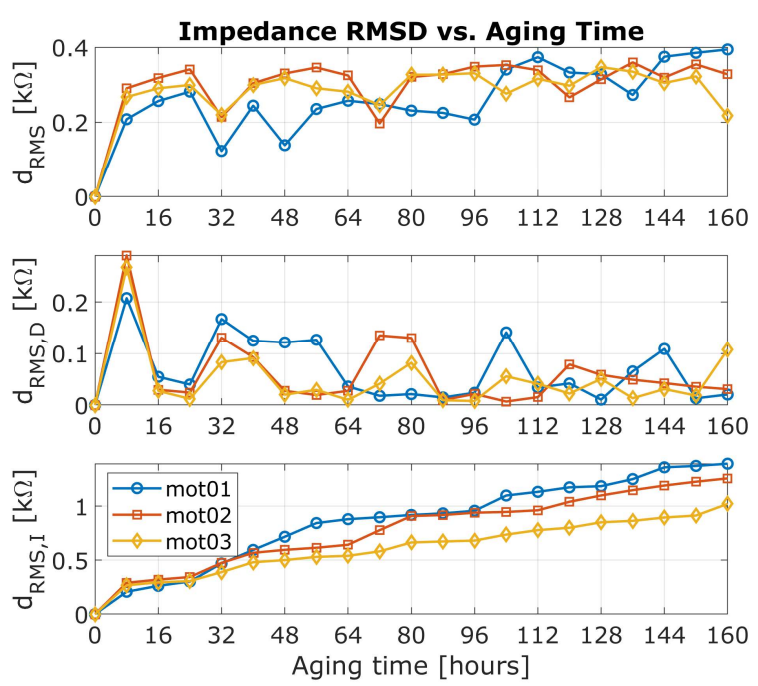

Fig. 11. Evolution of impedance deviation indices with aging time for the three motors under test.
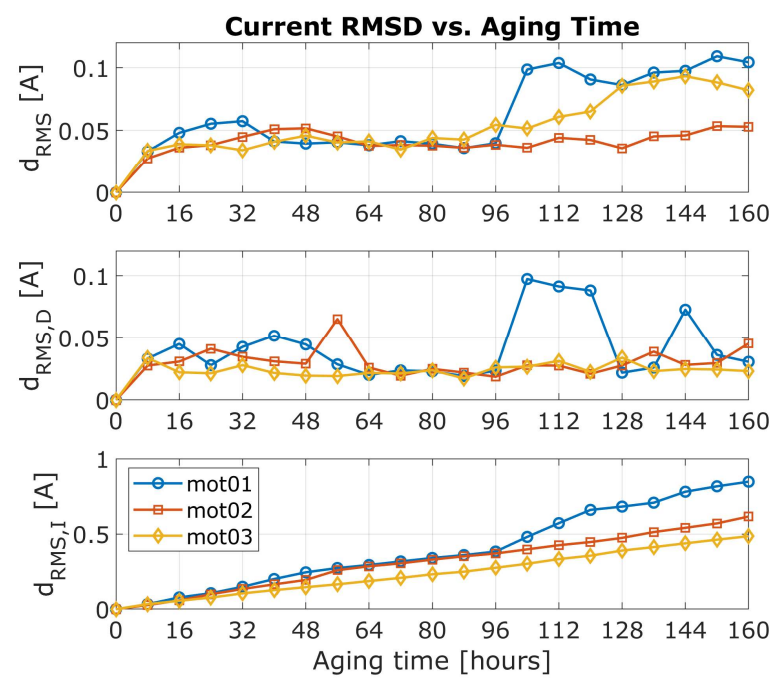

Fig. 12. Evolution of current deviation indices with aging time for the three motors under test.
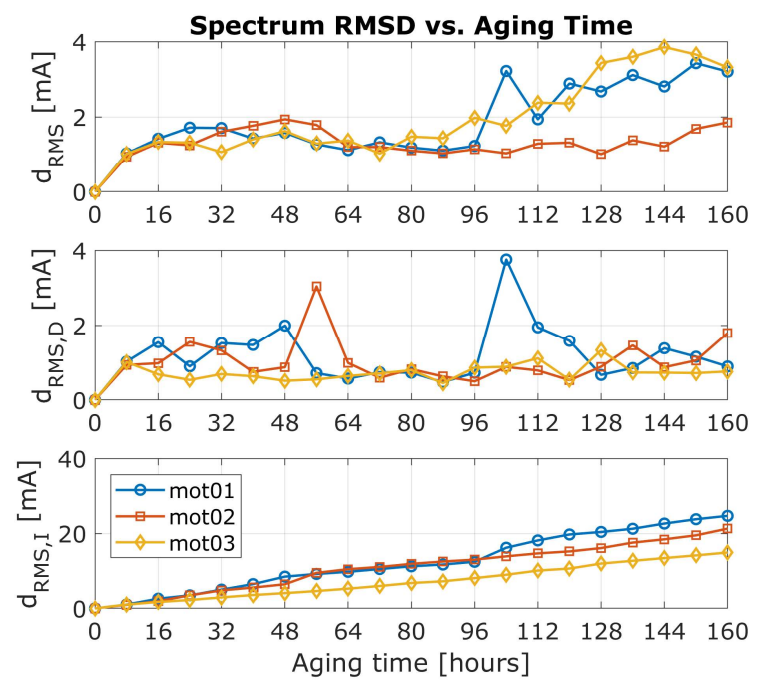

Fig. 13. Evolution of spectrum deviation indices with aging time for the three motors under test. 
sharp rise before settling around an almost constant value. For the MUT referred to as mot01 (blue lines in the figures) $d_{Z}$ initially settles around a value of about $0.2 \mathrm{k} \Omega$, then, after 96 hours of aging, it ramps up to a value around $0.35 \mathrm{k} \Omega$, similarly to mot02 (red line) and mot03 (yellow line). This step increase in the index could be due to an abrupt modification of the insulation state. From this result, one could wrongly infer a less intense aging progression for mot01 during the first 96 hours. However, if the differential deviation is considered, it can be noticed how mot01 undergoes more severe variations than the other two MUTs during the first 64 hours, leading to the highest integral deviation.

As it can be noticed by comparing Fig. 12 and Fig. 13, the trends of the current deviations and of the spectrum deviations are quite similar. The base deviations $d_{i}$ and $d_{Y}$ initially rise, with different rates for the three MUTs, to about the same value. After 96 hours, while the value for mot02 is almost constant, those for mot01 and mot03 sharply rise almost doubling. In particular, mot01 presents an abrupt variation similar, but more evident, to the one observed in the case of the impedance deviation. By looking at the differential deviations it is evident that mot02 and mot03 have a similar trend, but for the spike at hour 56. This reflects in the integral deviation value that is higher for mot02 despite the slope is the same. Therefore, mot02 presents a more advanced aging state than mot03, despite what observed in the base deviations. By comparing the base deviations of the three methods, it can be noticed that remarkable oscillations characterize the impedance deviation, making it difficult to recognize a clear trend for each motor. The trends of the current and spectrum deviations, instead, is more stable, and the aging phenomena can be easily detected. On the other hand, the integral deviations of the three indices feature very close results, since a distinct trend for each motor can be assessed.

Focusing on practical implementation aspects, advantages and drawbacks of the proposed approaches can be discussed. The impedance measure is essentially an off-line method since the motor must be disconnected from the system. Moreover, it requires expensive laboratory instrumentation (high-precision impedance analyzer) and can be time-consuming since three different winding connections have to be made, which require specific cabling of the MUT. The use of the high-frequency currents to detect aging progress can, instead, be performed during the motor start-up or included in self-commissioning procedures. In addition, it does not require expensive instrumentation, since the current measure could be made through commercial current transducers instead of the Rogowski coils, as demonstrated in [19]. As discussed before, the spectrum-based indices show similar performance with respect to those directly based on currents, which require less computational resources due to the absence of the FFT algorithm.

\section{CONCLUSIONS}

This paper presents the results of an electrical aging campaign on three AC motors to evaluate the effects on the insulation system produced by high-frequency, steep PWM voltage pulses. The novel approach consists in analyzing the effects on a whole stator winding insulation system by measuring macroscopic quantities, such as high-frequency impedances and currents. Three different indices are derived from the measures, each featuring an absolute, a differential, and an integral form. All the adopted indices show that the electrical aging progression is a phenomenon characterized by randomness since it can be interpreted as the cumulative effect of casual alterations happening in different points and with different rate within the insulation system. Among the different proposed solutions, the current-based indices are more easily applicable in a practical drive and give the best information about the aging progression. Regarding the index calculation, while the integral deviation performs better than the absolute deviation in describing the aging path that the motor undergoes, the differential deviation can give a useful indication about the intensity of the variations between two consecutive aging steps.

\section{REFERENCES}

[1] D. Gerada, A. Mebarki, N. L. Brown, C. Gerada, A. Cavagnino, and A. Boglietti, "High-speed electrical machines: technologies, trends, and developments," IEEE Trans. Ind. Electron., vol. 61, no. 6, pp. 29462958, June, 2014.

[2] N. Bianchi, S. Bolognani, and F. Luise, "Potentials and limits of highspeed PM motors," IEEE Trans. Ind. Appl., vol. 40, no. 6, pp. 1570-1578, Nov./Dec., 2004.

[3] A. S. Thomas, Z. Q. Zhu, R. L. Owen, G. W. Jewell, and D. Howe, "Multiphase flux-switching permanent-magnet brushless machine for aerospace application," IEEE Trans. Ind. Appl., vol. 45, no. 6, pp. 19711981, Nov./Dic., 2009

[4] H. Kim, M. W. Degner, J. M. Guerrero, F. Briz, and R. D. Lorenz, "Discrete-time current regulator design for AC machine drives," IEEE Trans. Ind. Appl., vol. 46, no. 4, pp. 1425-1435, July/Aug., 2010.

[5] D. Han, Y. Li, and B. Sarlioglu, "Analysis of SiC based power electronic inverter for high speed machines," in 2015 IEEE Applied Power Electronics Conference and Exposition (APEC'15), Charlotte, NC, USA, 2015, pp. 304-310.

[6] H. Zhang, L. M. Tolbert, and B. Ozpineci, "Impact of SiC devices on hybrid electric and plug-in hybrid electric vehicles," IEEE Trans. Ind. Appl., vol. 47, no. 2, pp. 912-921, Mar./Apr., 2011.

[7] C. Zoeller, M. A. Vogelsberger, T. M. Wolbank, H. Ertl, "Impact of SiC semiconductors switching transition speed on insulation health state monitoring of traction machines," IET Power Electronics, vol. 9, no. 15, pp. 2769-2775, Dec., 2016.

[8] B. Bolsens, K. De Brabandere, J. Van den Keybus, J. Driesen, and R. Belmans, "Transmission line effects on motor feed cables: terminator design and analysis in the Laplace-domain," in 2003 IEEE International Electric Machines and Drives Conference (IEMDC'03), Madison, WI, USA, 2003, pp. 1866-1872.

[9] A. F. Moreira, T. A. Lipo, G. Venkataramanan, and S. Bernet, "Highfrequency modeling for cable and induction motor overvoltage studies in long cable drives," IEEE Trans. Ind. Appl., vol. 38, no. 5, pp. 1297-1306, Sept./Oct., 2002.

[10] M. J. Melfi, "Low-voltage PWM inverter-fed motor insulation issues," IEEE Trans. Ind. Appl., vol. 42, no. 1, pp. 128-133, Jan./Feb., 2006.

[11] J. Rodríguez, J. Pontt, C. Silva, R. Musalem, P. Newman, R. Vargas, and $\mathrm{S}$. Fuentes, "Resonances and overvoltages in a medium-voltage fan motor drive with long cables in an underground mine," IEEE Trans. Ind. Appl., vol. 42, no. 3, pp. 856-863, May/June, 2006.

[12] A. Cavallini, D. Fabiani, and G. C. Montanari, "Power electronics and electrical insulation systems - part 1: phenomenology overview," IEEE Elect. Insul. Mag., vol. 26, no. 3, pp. 7-15, June, 2010.

[13] G. C. Stone, I. Culbert, E. A. Boulter, and H. Dhirani, "Evaluating insulation materials and systems," in Electrical Insulation for Rotating Machines: Design, Evaluation, Aging, Testing, and Repair, 2th ed. Piscataway, NJ, USA: Wiley-IEEE Press, 2014, ch. 2, sec. 1, pp. 49-54. 
[14] G. C. Montanari, "Bringing an insulation to failure: the role of space charge," IEEE Trans. Dielectr. Electr. Insul., vol. 18, no. 2, pp. 339-364, Apr., 2011.

[15] M. Kaufhold, H. Auinger, M. Berth, J. Speck, and M. Eberhardt, "Electrical stress and failure mechanism of the winding insulation in PWM-inverter-fed low-voltage induction motors," IEEE Trans. Ind. Electron., vol. 47, no. 2, pp. 396-402, Apr., 2000.

[16] P. Wang, A. Cavallini, and G. C. Montanari, "The influence of repetitive square wave voltage parameters on enameled wire endurance," IEEE Trans. Dielectr. Electr. Insul., vol. 21, no. 3, pp. 1276-1284, June, 2014.

[17] C. Petrarca, A. Maffucci, V. Tucci, and M. Vitelli, "Analysis of the voltage distribution in a motor stator winding subjected to steep-fronted surge voltages by means of a multiconductor lossy transmission line model," IEEE Trans. Energy Convers., vol. 19, no. 1, pp. 7-xxx, Mar., 2004.

[18] F. Gutfleisch, and L. Niemeyer, "Measurement and simulation of PD in epoxy voids," IEEE Trans. Dielectr. Electr. Insul., vol. 2, no. 5, pp. 729743, Oct., 1995.

[19] C. Zoeller, M. A. Vogelsberger, R. Fasching, W. Grubelnik, and T. M. Wolbank, "Evaluation and current-response-based identification of insulation degradation for high utilized electrical machines in railway application," IEEE Trans. Ind. Appl., vol. 53, no. 3, pp. 2679-2689, May/June, 2017.

[20] W. R. Jensen, E. G. Strangas, and S. N. Foster, "A method for online stator insulation prognosis for inverter-driven machines," IEEE Trans. Ind. Appl., vol. 54, no. 6, pp. 5897-5906, Nov./Dec., 2018.

[21] J. Yang, J. Cho, S. B. Lee, J. Yoo, and H. D. Kim, "An advanced stator winding insulation quality assessment technique for inverter-fed machines," IEEE Trans. Ind. Appl., vol. 44, no. 2, pp. 555-564, Mar./Apr., 2008.

[22] R. Leuzzi, V. G. Monopoli, L. Rovere, F. Cupertino, and P. Zanchetta, "Effects of electrical ageing on winding insulation in high-speed motors: analysis and modelling," in 2018 IEEE Energy Conversion Congress and Exposition (ECCE'18), Portland, OR, USA, 2018, pp. 6886-6892. 